\title{
VALVULOPLASTIA PERCUTÁNEA CON BALÓN DE VÁLVULA MITRAL DURANTE LA GESTACIÓN. REPORTE DE DOS CASOS EN UN HOSPITAL DE CUARTO NIVEL DE ATENCIÓN EN CALI, COLOMBIA, Y REVISIÓN DE LA LITERATURA
}

\section{Percutaneous balloon mitral valvuloplasty during pregnancy. Report of two cases in a level IV hospital in Cali, Colombia, and review of the literature}

Alejandro Colonia-Toro, MD, $\mathrm{MSc}^{1}$; María Fernanda Escobar-Vidarte, MD, $\mathrm{MSc}^{2}$; Jorge Velásquez-Noreña, MD'; Sara Sofía Escobar-González ${ }^{4}$; Angélica María Forero-Ladino, $M^{5}$

Recibido: septiembre 14/15 - Aceptado: noviembre 25/16

\section{RESUMEN}

Objetivo: describir la experiencia de dos casos de gestantes con estenosis mitral valvular, llevadas a valvuloplastia percutánea con balón y dilatación como alternativa terapéutica para contrarrestar los riesgos de colapso y deterioro materno-fetal y permitir la progresión del embarazo sin complicaciones cardiovasculares o el deterioro funcional, y realizar

1 Ginecoobstetra, Unidad de Alta Complejidad Obstétrica, Fundación Clínica Valle del Lili; magíster en Epidemiología, Universidad CES, Medellín (Colombia). alejocoloniat@yahoo.com

2 Ginecoobstetra, Universidad del Valle; maestría en Epidemiologia Clínica, Universidad de la Frontera, Chile; especialista en Cuidado Intensivo, Universidad del Valle, University of Pittsburgh. Jefe de la Unidad de Alta Complejidad Obstétrica, Fundación Clínica Valle del Lili; coordinadora de la Sección de Cuidado Crítico Obstétrico, Asociación Colombiana de Medicina Crítica y Cuidado Intensivo, Cali (Colombia).

3 Médico internista; especialista en Cardiología, Fundación Clínica Valle del Lili, Cali (Colombia).

4 Estudiante de pregrado, quinto año de Medicina, Universidad Icesi, Fundación Clínica Valle del Lili, Cali (Colombia).

5 Médico rural de investigaciones clínicas, servicio de Ginecología y Obstetricia, grupo de investigación en Unidad de Alta Complejidad Obstétrica, Fundación Clínica Valle del Lili, Cali (Colombia). una revisión de la literatura publicada sobre la realización del procedimiento en gestantes.

Materiales y métodos: se presentan dos casos de estenosis mitral severa y compromiso funcional asociado, de aparición en el segundo trimestre del embarazo, atendidos en una institución de cuarto nivel de complejidad, centro de referencia regional. Se manejaron por medio de valvuloplastia mitral con balón, con desenlaces clínicos maternos perinatales favorables. La revisión de la literatura se hizo a partir de los términos: "valvuloplastia", "estenosis mitral" y "embarazo". Se realizó una búsqueda en la base de datos Medline vía PubMed, Medes y Scopus. Se excluyeron los procedimientos descritos que estaban dirigidos al reparo valvular del feto. Los estudios se centraron en los cambios en las mediciones de la válvula, los resultados obstétricos y la seguridad. Resultados: se identificaron doce títulos. Los resultados obtenidos son comparables a los reportes de casos de estenosis de válvula mitral con procedimiento asistido por balón para dilatación de la 
válvula. En general, el procedimiento es exitoso, incrementa el área de la válvula mitral y reduce el gradiente de presión en la válvula. No obstante, existe el riesgo de desarrollar insuficiencia de válvula mitral tras la conducta, trombosis y muerte. Conclusión: la valvuloplastia percutánea con balón y dilatación como alternativa terapéutica es una opción por considerar en el manejo de la estenosis mitral en la gestante con deterioro de la clase funcional y el aumento durante el seguimiento de la presión en cuña pulmonar. Se requieren estudios controlados que validen los resultados de los estudios observacionales.

Palabras clave: estenosis de la válvula mitral, valvuloplastia con balón, embarazo.

\section{ABSTRACT}

Objective: To describe the experience of two cases of pregnant women with mitral stenosis taken to percutaneous balloon valvuloplasty and dilatation as a therapeutic option to counteract the risk of collapse and maternal and foetal deterioration, and to allow progression of the gestation without cardiovascular complications or functional impairment; and to conduct a review of the published literature regarding this procedure in pregnant women.

Materials and methods: Two cases of severe mitral stenosis and associated functional involvement of early onset during the second trimester of pregnancy, seen in a level IV regional referral centre. The two cases were managed with balloon mitral valvuloplasty, with favourable perinatal maternal outcomes. The review of the literature was done using the terms Valvulopasty, Mitral Stenosis and Pregnancy. A search was conducted in Medline via Pubmed and in the MEDES and SCOPUS databases. Procedures described for the performance of foetal valve repair were excluded. Studies were focused on the diagnostic findings and the course of the surgical procedure.

Results: Overall, 12 titles were identified. The results obtained are comparable with the case reports found in the literature on mitral valve stenosis using balloon-assisted procedures for valve dilatation. In general terms, the procedure is successful, increasing the area of the mitral valve and reducing the pressure gradient across the valve. However, there is a risk of mitral regurgitation, thrombosis and death following the procedure.

Conclusion: Percutaneous mitral valvuloplasty with balloon dilatation is a therapeutic option that may be considered for the management of mitral stenosis in pregnant women with functional class deterioration and increasing pulmonary wedge pressure during follow-up. Controlled studies are required in order to validate the results of observational studies.

Key words: Mitral valve stenosis, balloon valvuloplasty, pregnancy.

\section{INTRODUCCIÓN}

La estenosis mitral (EM) consiste en una obstrucción del flujo entre la aurícula y el ventrículo izquierdo causada por la anormalidad de la válvula. La enfermedad reumática es su principal causa. Reconocer la fisiopatología y las manifestaciones clínicas permite definir el grado de compromiso valvular, su efecto cardiovascular y la conducta terapéutica indicada en cada caso (1). Los métodos no invasivos como la ecocardiografía y la resonancia magnética, permiten el diagnóstico temprano y definir el manejo terapéutico que se debe seguir, con la intención de retardar el recambio valvular protésico (1). En la mujer no gestante, la valvuloplastia mitral percutánea con balón es el tratamiento de elección en estenosis mitral no calcificada, idealmente realizada previo a la concepción. Dentro de las indicaciones se destacan la falla cardiaca, New York Heart Association (NYHA) clase >II, regurgitación mitral y la estenosis subvalvular (2). Los resultados atribuibles al procedimiento son favorables y las complicaciones asociadas a su implementación son mínimas.

La estenosis mitral es la valvulopatia más común entre las gestantes (2). La serie de cambios hemodinámicos y funcionales que conlleva la instauración 
del embarazo, en particular durante el segundo trimestre, pueden llegar a ser, en la paciente con valvulopatia mitral, incompatibles con el progreso y la duración del mismo, poniendo en riesgo tanto de la vida de la madre como del feto (3).

Esta patología limita la capacidad funcional y la tolerancia de la materna a los eventos fisiológicos sucesivos que conlleva la gestación. La restricción de flujo del ventrículo izquierdo aumenta la cavidad auricular del mismo lado (inicialmente), la regurgitación del drenaje pulmonar aumenta la presión y la instauración de corto-circuito a este nivel. La obstrucción parcial del flujo sanguíneo en una válvula estenótica y el gradiente de presión en la válvula comprometida se afectan por el incremento fisiológico de la frecuencia cardiaca y del volumen latido durante el embarazo (4). Pacientes con gradiente transvalvular mayor de $101 \mathrm{~mm} / \mathrm{Hg}$, y estenosis valvular severa tienen repercusiones hemodinámicas como la alteración funcional de la materna, restricción del crecimiento fetal, óbito e hipertensión pulmonar $(2,5)$.

La falla cardiaca suele ser la complicación más frecuente debido al aumento de los gradientes de la válvula y disminución del área valvular, impidiendo el flujo libre de sangre a través de las cavidades y favoreciendo la resistencia sistémica. El volumen eyectado que vence la resistencia de la aorta, resulta insuficiente y termina por comprometer la perfusión materna y, en grados más avanzados, la circulación fetal (6). El trabajo de parto incrementa de 8 a $10 \mathrm{~mm} / \mathrm{Hg}$ la presión en cuña de la pulmonar (7).

La gran mayoría de las mujeres jóvenes, sin comorbilidades asociadas, con algún grado de estenosis, cursan asintomáticas y desconocen su condición clínica. Durante el embarazo, cuando el corazón se ve forzado a suplir el incremento metabólico y hemodinámico, aparecen los síntomas cardinales: disnea, cianosis y dolor torácico opresivo (8).

La falla cardiaca aguda durante el embarazo conlleva un riesgo 3 veces mayor de complicaciones obstétricas: la prematuridad (20-30\%), la restricción del crecimiento intrauterino (5-20\%) y la muerte fetal (1-3\%) (9). El deterioro funcional progresivo de la materna precipita la aparición de óbito fetal; su probabilidad, en estadios avanzados de la enfermedad (NYHA III y IV) aumenta en un $30 \%$, en ausencia de intervenciones médicas oportunas (10).

El cuidado de mujeres en embarazo con patología valvular requiere de un equipo multidisciplinario con experiencia. Idealmente, estas pacientes deberían iniciar su control antes de la concepción para mejorar y optimizar su estado hemodinámico durante la gestación, el parto y el puerperio (2). En la literatura se han reportado casos severos durante la gestación con clase funcional comprometida, asociado a la aparición de falla cardiaca o en quienes el manejo médico ha sido refractario (11).

Por esta razón, la intervención quirúrgica temprana en pacientes embarazadas se ha convertido en el tratamiento de elección cuando hay presencia de clase funcional NYHA III/IV, sintomáticas, que no responden al manejo médico conservador durante un abordaje inicial (12). La comisurotomía abierta como tratamiento en estas pacientes se ha asociado a la persistencia de los síntomas, mayor riesgo de infección, sangrado durante la cirugía, aparición de cambios valvulares de insuficiencia y riesgos fetales hasta ocho veces mayores con respecto a la valvuloplastia $(3,4)$.

No existen, a la fecha, indicaciones claras para realizar la valvuloplastia con balón durante el embarazo, sin embargo, la American Heart Association (AHA) la propone para: las pacientes sintomáticas, con estenosis moderada a severa e hipertensión pulmonar, así como pacientes con alto riesgo para cirugía abierta (9).

En nuestro país, la información sobre el uso de la valvuloplastia percutánea en mujeres gestantes con estenosis mitral con clase funcional NYHA III /IV es poca, por tanto, el objetivo de este estudio es describir dos casos de gestantes con estenosis mitral valvular llevadas a valvuloplastia percutánea con balón y dilatación como alternativa terapéutica en este subgrupo de población, y hacer una revisión 
de la literatura publicada sobre la realización del procedimiento en gestantes.

\section{PRESENTACIÓN DE LOS CASOS}

Lugar: Fundación Valle del Lili, Institución de cuarto nivel de complejidad, centro de referencia médica en el suroccidente colombiano, ubicada en la cuidad de Cali, Colombia.

\section{Caso 1}

Paciente de 25 años de edad, G1P0, con embarazo de 23,4 semanas por fecha de última menstruación, quien consulta a la institución por cuadro clínico de 10 días de evolución consistente en disnea de pequeños esfuerzos, asociada a la presencia de tos seca y ortopnea. Se le practicó ecocardiografía que reveló la presencia de hipertensión pulmonar severa, con presión sistólica de la arteria pulmonar de $115 \mathrm{~mm} /$ Hg y estenosis mitral grado III asociada, dilatación e hipertrofia del ventrículo derecho y aumento cavitario de la aurícula izquierda, por lo que es trasladada al servicio de alta complejidad obstétrica.

Fue valorada por el servicio de cardiología, con ecocardiografía transtorácica institucional que revela: severa dilatación de la aurícula izquierda, con ventrículo izquierdo de tamaño, espesor y contractilidad normales, fracción de eyección del ventrículo izquierdo de $79 \%$. Válvula mitral severamente engrosada, sin calcificaciones, con estenosis valvular severa, en ausencia de insuficiencia mitral asociada, con presencia de cavidades derechas dilatadas. Presión sistólica de la arteria pulmonar de $79 \mathrm{~mm} / \mathrm{Hg}$ y pequeño derrame pericárdico. El control fetoplacentario realizado a la fecha se encontraba dentro de los límites de normalidad para lo esperado en edad gestacional.

Durante la evolución clínica presenta persistente deterioro sostenido de la clase funcional, falla cardiaca derecha instaurada y se considera candidata a valvuloplastia mitral con balón. La decisión de la intervención por parte de cardiología fue expuesta en junta cardioquirúrgica, con el manejo interdisciplinario de la unidad de alta complejidad obstétrica en la institución.
Previo consentimiento informado es intervenida bajo anestesia general y local con lidocaína; por punción femoral bilateral se practicó valvuloplastia mitral percutánea, bajo control ecocardiográfico estricto, con gradiente transvalvular medio de válvula mitral de $20 \mathrm{~mm} / \mathrm{Hg}$, que luego de avanzar y dilatar balón INOUE hasta $28 \mathrm{~mm}$, disminuye a 11 $\mathrm{mm} / \mathrm{Hg}$. Ventriculografía izquierda revela mejoría del llenado ventricular y ausencia de insuficiencia valvular mitral significativa, con monitoreo fetal y materno continuo. El ecocardiograma transesofágico de control confirma la presencia de cavidades izquierdas dilatadas, con fracción de eyección conservada de $65 \%$, hipertensión pulmonar persistente de $74 \mathrm{~mm} / \mathrm{Hg}$, con estenosis mitral severa y gradiente valvular medio de $12 \mathrm{~mm} / \mathrm{Hg}$.

Continuó el manejo materno-fetal, con monitoreo permanente en la unidad de cuidados intensivos, donde se suministra inicialmente betabloqueador a bajas dosis (metoprolol $100 \mathrm{mg} /$ día) para el control de la frecuencia cardiaca y con el fin de favorecer el tiempo de llenado en diástole. Requirió adicionalmente manejo con diurético endovenoso (furosemida $20 \mathrm{mg} /$ día) por la aparición de edema en miembros inferiores y signos clínicos de sobrecarga de volumen.

Durante la estancia hospitalaria su evolución resultó favorable, con mejoría crítica de su clase funcional, independiente del suministro de oxígeno. La paciente recibe el alta médica 3 días después del procedimiento, sin complicaciones asociadas al momento y tras la valoración pertinente y consensuada de ambos servicios tratantes. Acude nuevamente al servicio con 29,6 semanas de gestación, asintomática $\mathrm{y}$ sin deterioro de la clase funcional. Se realiza un nuevo ecocardiograma que reporta: aurícula izquierda dilatada, con ventrículo izquierdo de dimensiones, grosor y contractilidad conservadas; fracción de eyección del ventrículo izquierdo del $65 \%$; estenosis mitral severa con gradiente medio de $17 \mathrm{~mm} / \mathrm{Hg}$ e insuficiencia tricúspide 2/4; las cavidades derechas se encontraban severamente dilatadas, pero su contractilidad se mantenía, la presión sistólica de la arteria pulmonar presentaba un ascenso a $117 \mathrm{~mm} / \mathrm{Hg}$. 
Debido al alto riesgo de complicaciones maternas y fetales en el último trimestre del embarazo, en función de la carga hemodinámica y los nuevos riesgos de edema pulmonar, falla y colapso cardiopulmonar tras las cifras de presión pulmonar, se decide completar las 30 semanas de embarazo para garantizar la viabilidad del feto. Con 30,2 semanas de gestación, es llevada a cesárea sin complicaciones aparentes durante el procedimiento, se obtiene recién nacido femenino de $1600 \mathrm{gy} 40 \mathrm{~cm}$, con APGAR 8/10 a los 5 min y favorable evolución clínica a cargo del servicio de UCI neonatal. Durante el puerperio la paciente persiste asintomática y dentro de parámetros de normalidad, es dada de alta.

\section{Caso 2}

Paciente G3P1A1 con embarazo de 14 semanas, quien consultó a la institución por cuadro de 2 años de evolución consistente en disnea progresiva de moderados esfuerzos, asociada a la presencia de soplo sistólico. Se realizó ecocardiograma que reportó esclerosis valvular aórtica sin estenosis, con insuficiencia moderada; enfermedad valvular mitral con estenosis e insuficiencia moderada; insuficiencia tricúspide leve a moderada con hipertensión pulmonar moderada; dilatación de la aurícula izquierda de 43 mm y fracción de eyección del 66\%. Fue valorada por cardiólogo quien recomendó continuar la gestación y consideración de parto por cesárea con profilaxis para endocarditis bacteriana.

La paciente consulta nuevamente, con embarazo de 18 semanas y cuadro consistente en dificultad respiratoria con compromiso hemodinámico y sospecha de neumonía adquirida en la comunidad. Es hospitalizada para manejo endovenoso con ceftriaxona y toma sucesiva de paraclínicos, donde la radiografía de tórax reporta infiltrados bibasales y borramiento de ángulo costo-diafragmático izquierdo.

$\mathrm{Al}$ ser valorada por medicina interna agregan claritromicina al tratamiento y realizan ecocardiograma que evidencia estenosis mitral severa con área de 0,97, hojuela posterior gruesa y rígida, insuficiencia aórtica grado I-II, insuficiencia tricúspidea grado II, presión sistólica pulmonar $60 \mathrm{~mm} / \mathrm{Hg}$ y cavidades derechas dilatadas. Se considera paciente con edema pulmonar asociado a válvula disfuncional y sospecha de neumonía, razón por la cual se considera paciente con diagnóstico de estenosis mitral severa.

Se realiza ecocardiograma transesofágico que reportó obstrucción severa mitral con gradiente medio de $22 \mathrm{~mm} / \mathrm{Hg}$ sin insuficiencia, sin calcificación ni retracción del aparato valvular o subvalvular; fracción de eyección (FE) 60-65\%, presión sistólica de la arteria pulmonar $72 \mathrm{~mm} / \mathrm{Hg}$ con insuficiencia tricúspidea leve. Para descartar la presencia de endocarditis se realizaron dos hemocultivos que fueron negativos.

En junta multidisciplinaria con cardiología no invasiva, hemodinámica, cirugía cardiovascular y alta complejidad obstétrica se decidió realizar valvuloplastia con balón. Se realizó ecografía obstétrica con perfil biofísico normal. Previo consentimiento informado de la paciente es llevada al servicio de hemodinámica donde se realiza el procedimiento. Tras anestesia espinal con lidocaína se lleva a cabo inserción percutánea de catéter femoral derecho de alto flujo, paso posterior de guía con catéter inserto con balón de INOUE que se dilata al entrar a cavidad de válvula aurícula-ventricular hasta $3 \mathrm{~cm}$. Se realiza ecografía transesofágica simultánea que evidencia la disminución inmediata del gradiente transmitral a $10 \mathrm{~mm} / \mathrm{Hg}$. Se retira nuevamente el catéter implantado y la guía, con presión sobre el área de punción, procedimiento que culmina sin complicaciones.

Es trasladada a la Unidad de Alta Complejidad Obstétrica (UACO) para monitoreo fetal y materno continuo. El ecocardiograma de control reveló insuficiencia mitral moderada a severa; insuficiencia tricúspidea leve; presión sistólica de la arteria pulmonar de $46 \mathrm{~mm} / \mathrm{Hg}$; foramen oval permeable con cortocircuito pequeño de derecha a izquierda. Permanece en UACO en manejo con betabloqueador (metoprolol) y profilaxis antitrombótica. Al cuarto día posoperatorio es dada de alta sin signos de falla cardiaca y continúa con un estricto control 
ambulatorio interdisciplinario con cardiología y alta complejidad obstétrica.

A las 38 semanas de gestación consultó por inicio de trabajo de parto, previo control ecocardiográfico sin modificaciones. Se realiza atención monitorizada del trabajo de parto con analgesia obstétrica, con expulsivo instrumentado con espátulas de Velasco, obteniéndose un recién nacido de 2290 g, talla de 45 cm y APGAR 8/9. Se realizó manejo farmacológico del tercer periodo del parto con carbetocina.

En el posparto inmediato la paciente inicia con polipnea, disnea y saturación de oxígeno de 88; estertores en ambas bases pulmonares; al considerar edema pulmonar por sobrecarga de volumen secundario al retorno del shunt fisiológico del embarazo se decide traslado a la UCI y se inicia tratamiento con nitroglicerina, morfina, furosemida y ventilación mecánica no invasiva. La paciente evoluciona satisfactoriamente y es dada de alta al tercer día con enalapril, metoprolol y controles periódicos por cardiología, sin otras complicaciones referidas.

Durante control anual el ecocardiograma reportó FE $75 \%$, válvula mitral engrosada con leve estenosis, insuficiencia mitral severa e insuficiencia aórtica leve; cavidades derechas normales; presión pulmonar sistólica $30 \mathrm{~mm} / \mathrm{Hg}$; clase funcional I.

\section{MATERIALES Y MÉTODOS}

Se llevó a cabo una búsqueda de la literatura en las bases de datos Medline vía PubMed, Medes y Scopus, con los términos de búsqueda: "valvuloplastia”, "estenosis mitral” y "embarazo". Se buscaron reportes de casos, reportes de series de casos, cohortes, guías de práctica clínica y se revisó la bibliografía citada en el artículo, sin límite de tiempo de la búsqueda y los idiomas. Se descartaron cartas al editor. Los estudios se centraron en los cambios en las mediciones de la válvula, los resultados obstétricos y la seguridad.

Aspectos éticos. Las pacientes dieron autorización para la publicación de su caso y se garantizó la confidencialidad de la información y su privacidad.

\section{RESULTADOS}

De un total de 740 títulos se identificaron 12 títulos relativos al uso de la valvuloplastia mitral percutánea con balón en mujeres gestantes, de los cuales 6 correspondieron a reporte de casos $(4,13,14-17)$ y 6 a series de casos $(8,11,18-21)$. Los estudios fueron realizados en Brasil $(8,11,17)$, Portugal (4), Sur África (14), Túnez (21), Irán (20), México (16), India (13), Francia (15) y Estados Unidos (14, 19).

Ben Farhat et al. (21) informan una serie de casos de 44 gestantes sometidas al procedimiento entre 1990 y 1996, con una edad gestacional promedio de 26 semanas; 4 pacientes habían sido sometidas previamente a comisurotomía por balón. Estado funcional NYHA III y IV. Describen que no hubo fallas en el procedimiento. El área de la válvula mitral incrementó $1,07(0-21)$ a 2,32 (0-36) $\mathrm{cm}^{2}$. El parto fue vaginal a término en 42 de las pacientes y 2 pacientes por cesárea. Las complicaciones fueron: regurgitación mitral en 2 pacientes, una trombosis venosa y 1 daño valvular que requirió reemplazo de la válvula.

Andrade et al. (8) informan los resultados de 39 mujeres con estenosis mitral de origen reumático, que fueron sometidas al procedimiento durante el embarazo con un promedio de 26 años, estadio NYHA III-IV. La cirugía fue exitosa, en ningún caso se necesitó reintervención. El área valvular media pasó de 0,84 a 2,14 $\mathrm{cm}^{2}$. El $77 \%$ de los partos fueron por cesárea, se presentaron un aborto, un óbito fetal y la muerte de dos gemelos por insuficiencia respiratoria del RN. No hubo complicaciones.

Esteves (11) et al. realizaron un estudio descriptivo, en el cual incluyeron 71 pacientes gestantes entre $27+/-6$ años y rango de edad gestacional entre 6-34 semanas, que fueron intervenidas con valvuloplastia percutánea con balón. Se trataba de pacientes NYHA grado III o IV, con falla cardiaca y dosis máxima de medicamentos. Posterior al procedimiento presentaron disminución significativa en la presión media de la aurícula izquierda, presión diastólica mitral y 
presión media de la arteria pulmonar. El promedio de edad gestacional al finalizar el embarazo fue 38 +/- 1,2 semanas, una paciente presentó aborto espontáneo, 20 pacientes tuvieron parto vaginal y las demás cesárea, se presentó un mortinato, 4 pacientes tuvieron gemelos y 9 pacientes tuvieron neonatos pretérmino. Dos pacientes presentaron trombo-embolismo pulmonar no fatal (11).

Patel et al. (18) informan el uso de la técnica en 19 pacientes; no refieren el estado de clase funcional antes de la cirugía. Utilizaron el catéter INOUE en 18 pacientes. Reportan que el gradiente de la válvula mitral disminuyó de 17,9 \pm 6,2 a 5,9 \pm 2,4 $\mathrm{mm} / \mathrm{Hg}$ y un incremento en el área de la válvula mitral desde $0,8 \pm 0,2$ a 1,7 $\pm 0,2 \mathrm{~cm}^{2}$. Todas las pacientes tuvieron mejoría de la clase funcional al menos en una categoría, 18 pacientes tuvieron parto normal, una paciente presentó regurgitación mitral moderada $(3+)$ como complicación.

Abouzied et al. (19) informan el uso del procedimiento en 16 gestantes clase funcional III y IV. Reportan que el área de la válvula mitral se incrementa de $0,9 \pm 0,3$ a 1,8 $\pm 0,3 \mathrm{~cm}^{2}$. El gradiente de la válvula mitral disminuyó de $23 \pm 7$ a $6 \pm 3$ $\mathrm{mm} / \mathrm{Hg}$. También informan reducción de la presión en la aurícula izquierda, en la arteria pulmonar y mejoría de la clase funcional. Un total de 11 niños nacieron por parto vaginal, un total de 14 nacieron a una edad gestacional mayor a 37 semanas y 2 presentaron parto pretérmino. No informan complicaciones.

Salehi et al. (20) informan que sometieron a 24 pacientes al procedimiento por EM severa; sin embargo, perdieron 14 pacientes en el seguimiento. Indican que la tasa de éxito fue del $100 \%$, pero 2 de los 10 recién nacidos fallecieron por falla cardiaca, aunque no es claro si de la madre o del neonato.

En cuanto a la seguridad, un reporte de caso informa tromboembolismo coronario durante el procedimiento que fue adecuadamente manejado (13), y otro reporta un taponamiento cardiaco por perforación cardiaca durante el procedimiento, con manejo adecuado y evolución favorable (17). Este autor informa otras posibles complicaciones asociadas al procedimiento, además de las mencionadas, tales como: muerte, shock y embolismo sistémico.

Por último, un reporte de caso describe la realización del procedimiento con anestesia intravenosa (14), y otro discute la protección fetal y de la madre de la radiación durante el procedimiento (15).

\section{CONCLUSIONES}

La valvuloplastia percutánea con balón es una alternativa que parece segura y eficaz en el manejo de pacientes grávidas con diagnóstico de estenosis mitral moderada a severa y clase funcional NYHA III/IV asociada a la aparición de hipertensión pulmonar. Las complicaciones más frecuentes son la regurgitación mitral, el tromboembolismo y la ruptura cardiaca. Se requieren estudios con mayor rigor metodológico que permitan hacer una evaluación más adecuada de su efectividad y seguridad.

\section{REFERENCIAS}

1. Cubides C. Fisiopatología de la falla cardiaca. En Castro H, Cubides C. Editores. Falla Cardiaca, diagnóstico y manejo actual. Manizales: Editorial Blanecolor; 2004. p. 15-32.

2. Tsiaras S, Poppas A. Mitral valve disease in pregnancy: outcomes and management. Obstet Med. 2009;2:6-10.

3. Siu SC, Colman JM, Sorensen S, Smallhorn JF, Farine $\mathrm{D}$, Amankwah KS, et al. Clinical Investigation and Reports. Adverse neonatal and cardiac outcomes are more common in pregnant women with cardiac disease. Circulation. 2002;105:2179-84.

4. Salomé N, Díaz CC, Ribeiro J, Goncalves M, Fonseca C, Ribeiro VG. Ballon mitral valvuloplasty during pregnancy-our experience. Rev Port Cardiol. 2002;21:1437-44

5. Gei A, Hankins G. Cardiac disease and pregnancy. Obstet Gynecol Clin North Am. 2001;28:465-512.

6. Hameed A, Karaalp IS, Tummala PP, Wani OR, Canetti M, Akhter MW, et al. The effect of valvular heart disease on maternal and fetal outcome of pregnancy. J Am Coll Cardiol. 2011;893-9. 
7. Reimold SC, Rutherford JD. Valvular heart disease in pregnancy. N Engl J Med. 2003;349:52-9.

8. Andrade J, Maldonado M, Pontes S, Elmec AR, Sousa JE. Papel de la valvuloplastia por catéter-balón durante el embarazo en mujeres portadoras de estenosis mitral reumática. Rev Esp Cardiol. 2001;54:573-9.

9. American College of Cardiology, American Heart Association Task Force on Practice Guidelines, Society of Cardiovascular Anesthesiologists, Bonow RO, Carabello BA, Chatterjee K, et al. ACC/AHA 2006 guidelines for the management of patients with valvular heart disease: a report of the American College of Cardiology/American Heart Association Task Force on Practice Guidelines. J Am Coll Cardiol. 2006;48:e1-148.

10. Brady K, Duff P. Rheumatic heart disease in pregnancy. Clin Obstet Gynecol. 1989;32:21-40.

11. Esteves CA, Muñoz JS, Braga S, Andrade J, Meneghelo Z, Gomes N, et al. Immediate and long term follow up of percutaneous ballon mitral valvuloplasty in pregnant patients with rheumatic mitral stenosis. Am J Cardiol. 2006;98:812-6.

12. Weiss BM, von Segesser LK, Alon E, Seifert B, Turina MI. Outcome of cardiovascular surgery and pregnancy: A systematic review of the period 1984-1996. Am J Obstet Gynecol. 1998;1643-53.

13. Chikkabasavaiah N, Rajendran R. Percutaneous Coronary Intervention for Coronary Thromboembolism during Balloon Mitral Valvuloplasty in a Pregnant Woman. Heart Lung Circ. 2016;25:e29-31. Doi: 10.1016/j.hlc.2015.06.824.

14. Apple L, Gupta D, Okumura M, Wang H. Percutaneous balloon mitral valvuloplasty in a pregnant patient under minimally invasive intravenous anesthesia. Middle East J Anaesthesiol. 2012;21:627-9.

15. Leroux L, Peragallo N, Dijos M, Murman M, Casassus F, Coste P, et al. Percutaneous mitral commissurotomy during pregnancy: With or without pelvic lead apron use? Int J Cardiol. 2015;188:70-2. Doi: 10.1016/j. ijcard.2015.04.058.

16. Iñigo CA, Solórzano CJ, Delgado G, Valdivia SO, Gómez G. Percutaneous mitral valvuloplasty with Inoue balloon and pregnancy. Ginecol Obstet Mex. 1998;66:435-9.

17. Seca L, Costa M, Quintal N, Marques AM. Percutaneous mitral valvuloplasty complicated by cardiac tamponade in a pregnant patient. Arq Bras Cardiol. 2008;91:e45-7.

18. Patel JJ, Mitha AS, Hassen F, Patel N, Naidu R, Chetty $\mathrm{S}$, et al. Percutaneous ballon mitral valvotomy in pregnant patients with tight pliable mitral stenosis. Am Heart J. 1993;125:1106-9.

19. Abouzied AM, Al Abbady Mi, Al Gendy MF, Magdy A, Soliman H, Faheem F, et al. Percutaneous balloon mitral commissurotomy during pregnancy. Angiology. 2001;52:205-9.

20. Salehi R, Aslanabadi N, Taghavi S, Pourafkari L, Imani S, Goldust M. Percutaneous balloon mitral valvotomy during pregnancy. Pak J Biol Sci. 2013;16:198-200.

21. Ben Farhat M, Gamra H, Betbout F, Maatouk F, Jarrar M, Addad F, et al. Percutaneous balloon mitral commissurotomy during pregnancy. Heart. 1997;77:564-7. 